\title{
Acesso, prática educativa e empoderamento de pacientes com doenças crônicas
}

\author{
Access, educational practice \\ and empowerment of patients with chronic diseases
}

Patricia da Silva Taddeo ${ }^{1}$

Kilma Wanderley Lopes Gomes ${ }^{1}$

Andrea Caprara ${ }^{1}$

Annatália Meneses de Amorim Gomes ${ }^{1}$

Giselle Cavalcante de Oliveira ${ }^{1}$

Thereza Maria Magalhães Moreira ${ }^{1}$

${ }^{1}$ Departamento de Saúde Publica, Centro de Ciências da Saúde, Universidade Estadual do Ceará. Av. Paranjana 1700, Itaperi. 60740-000 Fortaleza CE. patriciataddeo@hotmail.com

\begin{abstract}
Empowerment is a process whereby people achieve mastery over their lives, acquiring knowledge to make decisions about their health. Chronic diseases are increasingly common in the health service, requiring the attention of health professionals and empowering those affected by them. The scope of this study was to analyze the perceptions of users with chronic diseases and monitored by the Family Health Program with respect to empowerment. This was a qualitative study in which open interviews and systematic observations were conducted and NVIVO software was used for data analysis. Users indicate the existence of some geographical barriers in access to health, which caused fatigue, discouragement and low continuity of care. It was noted that adherence and practices of care were linked to differentiated service, based on trust and respect for the wishes of users. This includes healthcare guidance and education as key elements to encourage the practice of self-care. It is necessary to restructure the conduct of health professionals involved in the Family Health Strategy because its function is to promote health in a logical intersectoral and interdisciplinary manner.
\end{abstract}

Key words Chronic diseases, Empowerment, Family health
Resumo O Empoderamento é um processo pelo qual as pessoas adquirem o dominio sobre suas vidas, apreendendo conhecimento para tomar decisões acerca de sua saúde. Cada dia mais comuns, as doenças crônicas estão presentes no serviço de saúde, requerendo atenção dos profissionais de saúde e empoderamento dos acometidos por elas. Este estudo teve por objetivo analisar as percepções dos usuários com doenças crônicas, em acompanhamento na Estratégia Saúde da Família sobre o empoderamento. Tratou-se de uma pesquisa qualitativa na qual foram realizadas entrevistas abertas e observação sistemática. A análise dos dados se deu com a utilização do software NVIVO. Os usuários apontam a existência de algumas barreiras geográficas no acesso à saúde gerando fadiga e falta de estímulo, e ocasionando baixa continuidade do tratamento. Observou-se que a adesão e a prática do cuidado estão intimamente ligadas ao atendimento diferenciado, baseado na confiança e no respeito aos anseios dos usuários. Estes consideram a orientação e a educação em saúde como elementos principais para incentivar a prática do cuidado de si. Torna-se necessário reestruturar a conduta dos profissionais inseridos na Estratégia Saúde da Família, uma vez que tem como função a promoção da saúde, em uma lógica intersetorial e interdisciplinar. Palavras-chave Doenças crônicas, Empoderamento, Saúde da Família 


\section{Introdução}

Cada vez mais comuns em nossa sociedade, as doenças crônicas estão mais presentes nos serviços de saúde, requerendo atenção redobrada dos seus profissionais.

A condição crônica pode ser considerada como uma experiência de vida permanente, causada por doenças que acarretam perdas e disfunções, além da alteração no quotidiano. Essa permanência causa estresse devido à alteração da imagem corporal, necessidade de adaptação social e psicológica, além de mudança na expectativa de vida ${ }^{1}$.

As Doenças Crônicas Não Infecciosas (DCNI) exercem papel importante no perfil atual de saúde das populações humanas e a discussão acerca desse tema vem conquistando cada vez mais espaço no sentido de sermos capazes de manter a integridade, a independência e a autonomia dos indivíduos acometidos. Estimativas da Organização Mundial de Saúde (OMS) apontam que as DCNI já são responsáveis por $58,5 \%$ de todas as mortes ocorridas no mundo e por $45,9 \%$ da carga global de doença².

O sistema de saúde encontra-se focado na doença aguda ao invés de doenças crônicas, o que culmina em pacientes desinformados e despreparados para realizar o autocuidado. Os planejamentos das visitas são breves e não há certeza de que as necessidades agudas e crônicas serão eficientemente atendidas ${ }^{3}$.

Para que haja melhoria dos cuidados crônicos, é necessária uma articulação entre a organização fornecedora e a comunidade, utilizandose os recursos comunitários ${ }^{3}$. O serviço de saúde precisa de reorganização pela implantação de uma rede integrada de serviços para, assim, garantir o cuidado contínuo ${ }^{4}$. Sousa ${ }^{5}$, faz reflexões sobre mudanças de paradigma e concepções do atual modelo ainda focado na doença, pelo Modelo de Cuidados Crônicos, que tem na abordagem à pessoa, com seus problemas diversos, a complementaridade com os profissionais de saúde, a comunidade, o paciente e sua família.

Os cuidados crônicos ocorrem dentro de três esferas interdependentes: a comunidade, com suas políticas e recursos; o sistema de saúde; e a organização fornecedora, seja uma pequena clínica, uma rede frouxa de práticas sanitárias ou um sistema domiciliar integrado ${ }^{3}$. A filosofia do modelo de cuidados crônicos prega a melhoria na qualidade do atendimento aos pacientes com doenças crônicas, por meio de uma abordagem pró-ativa, capaz de prever e antecipar possíveis complicações e exacerbações da doença e apre- sentar o envolvimento dos pacientes, de sua família e da comunidade, além de uma forte integração entre cuidados primários e secundários, da utilização de programas de cuidados e de condutas baseadas em evidências ${ }^{4}$.

O empoderamento é um processo educativo destinado a ajudar os pacientes a desenvolver conhecimentos, habilidades, atitudes e autoconhecimento necessário para assumir efetivamente a responsabilidade com as decisões acerca de sua saúde ${ }^{6}$. Pacientes mais informados, envolvidos e responsabilizados (empoderados), interagem de forma mais eficaz com os profissionais de saude tentando realizar ações que produzam resultados de saude .

Este estudo teve como objetivo analisar as percepções dos usuários com doenças crônicas, em acompanhamento na Estratégia Saúde da Família sobre o empoderamento.

\section{Metodologia}

O estudo qualitativo descritivo foi desenvolvido em Fortaleza-Ceará-Brasil, com a apreensão do autocuidado a partir das percepções dos pacientes com doenças crônicas sobre empoderamento. O campo de pesquisa foi de três Unidades de Saúde da Família. Os critérios para sua seleção foram: 1) ter equipe mínima completa de Saúde da Família (Médico, Enfermeiro, 6-12 Agente Comunitário de Saúde, Auxiliar de Enfermagem); 2) possuir Núcleo de Apoio à Saúde da Família (NASF). Os dados foram coletados em entrevistas abertas e observação sistemática com pacientes.

Foram selecionados intencionalmente para participar da pesquisa dez usuários com diabetes e/ou hipertensão atendidos na Estratégia Saúde da Família em cada uma das USF estabelecidas, totalizando trinta usuários, todos eram participantes de grupo de educação em saúde desenvolvido pela equipe (quando havia) ou outra atividade educativa na comunidade e assistidos em visita domiciliar.

No tocante à observação, a pesquisadora participou de algumas atividades que integraram o itinerário terapêutico do paciente com doença crônica, observando e registrando no diário de campo os seguintes cenários: visita domiciliar, consulta individual, consulta coletiva e atividade em grupo, sala de espera e triagem. Esta observação permitiu captar informações que foram complementares às entrevistas.

Os dados foram analisados adotando como ferramenta para organização e análise dos da- 
dos o software Qualitative Solutions Research Nvivo (QSR) para análise qualitativa de dados, desenvolvido pela Universidade de La Trobe, Melbourne, Austrália8.

Inicialmente todos os dados foram transcritos para o software na forma de documentos com a extensão ${ }^{*}$.rtf (rich text format) disponível no Microsoft Word. E para que o processo de recorte e agrupamento fosse facilitado todas as linhas do texto foram numeradas.

Sendo assim, os passos realizados na aplicação do N-vivo foram: 1) codificação ou categorização - representação de uma categoria ou ideia abstrata onde é possível armazenar sua definição e que no N-vivo nomeia-se de "nós"; 2) conceituação das Categorias; 3) Agrupamento; e 4) análise dos dados.

$\mathrm{Na}$ realização desta pesquisa, obedeceu-se à Resolução 196/96 do Conselho Nacional de Saú$\mathrm{de}^{9}$, que regulamenta os aspectos ético-legais da pesquisa com seres humanos, mediante a aprovação do projeto guarda-chuva pelo Comitê de Ética em Pesquisa da Universidade Estadual do Ceará.

Por fim, ressalte-se que a coleta de dados foi realizada no período de uma epidemia de dengue, onde os postos de saúde estavam totalmente mobilizados em dar assistência aos doentes agudos para evitar a superlotação dos hospitais.

Além disso, no mesmo período houve uma greve dos profissionais, o que limitou o número de atendimentos nos postos e, consequentemente, dificultou localizar pacientes com doenças crônicas. Também encontramos empecilhos em acompanhar os grupos de educação e visita domiciliar em algumas regionais, pois os mesmos foram suspensos devido a paralisação dos profissionais.

Por se tratar de uma pesquisa com usuários da ESF em alguns postos não havia um local adequado para a realização das entrevistas, muitas vezes tendo que realizá-las na fila do atendimento. Isso dificultou um pouco na concentração deles, além de dificultar a gravação por conta do ruído externo.

\section{Resultados e discussão}

Dentre as categorias elaboradas teceremos discussão em relação às barreiras ao acesso e às possibilidades de enfrentamento pelo empoderamento, $\mathrm{O}$ papel do empoderamento na relação profissional-paciente e a prática educativa e empoderamento na relação de cuidado.

\section{Barreiras ao acesso e possibilidades de enfrentamento pelo empoderamento}

Os pacientes com doenças crônicas necessitam de acompanhamento constante, porém a realidade dos usuários pesquisados aponta a existência de barreiras geográficas que impossibilitam sua locomoção, o acesso aos serviços de saúde e, por consequência, restringem as possibilidades da adesão terapêutica.

$\mathrm{O}$ acesso é apresentado como um dos elementos do sistema de saúde, dentre aqueles ligados à organização dos processos de trabalho, que se refere à entrada nele, recebimento de cuidados subsequentes que garantam a continuidade do tratamento ${ }^{10}$, sendo a garantia de acesso resolutivo, em tempo oportuno e com qualidade, às ações e serviços o maior desafio do Sistema Único de Saúde-SUS ${ }^{11}$.

Percebeu-se neste estudo que a maior dificuldade está na distância entre o domicílio e a Unidade de Saúde da Família. Como dependem do transporte público para sua locomoção, muitas vezes não conseguem chegar tão próximo do posto, pois a organização da malha viária dificulta a acessibilidade, e o usuário realiza longas caminhadas, que os leva à fadiga agravada pela condição de vulnerabilidade e à falta de estímulo em sequenciarem o tratamento.

Com eles carregam apenas os anseios e a esperança de conseguir um atendimento diferenciado e humanizado, o que contraria as evidências científicas as quais sugerem que pacientes com condições crônicas obtém melhor resultado clínico quando recebem tratamento efetivo dentro de um sistema integrado de cuidado, o que inclui o suporte ao autocuidado e acompanhamento regular $^{12}$; os princípios do Modelo de Cuidado Crônico ${ }^{3}$; e as diretrizes da Política Nacional de Humanizaçao ${ }^{13}$.

É importante considerar que na perspectiva da saúde coletiva, as condições de desigualdade persistentes em dada realidade constituem iniquidades a serem enfrentadas visando diminuir as vulnerabilidades a que certos grupos populacionais estão expostos, e que resultam de determinantes sociais da saúde como níveis de escolaridade e de renda, condições de habitação, transporte, entre outros ${ }^{11}$. O empoderamento aparece como peça-chave na promoção da saúde desde a Carta de Ottawa em 1986, entendido como processo de capacitação dos indivíduos e comunidades para assumirem maior controle sobre os fatores pessoais, socioeconômicos e ambientais que afetam a saúde ${ }^{14}$. Está relacionado também com 
a equidade, as condições reais que as pessoas têm a seu favor para obterem condições de vida saudável, a comunicação e a participação social.

O empoderamento requer o diálogo entre o profissional da saúde e a comunidade, de modo a identificar necessidades que levem a uma intervenção em virtude do que a comunidade precisa, contando com seu protagonismo na fixação da agenda da promoção da saúde ${ }^{15}$. Nesse sentido, ações intersetoriais, envolvimento do controle social e escuta às necessidades dos usuários podem ser articuladas no contexto, visando estabelecer compromissos e projetos para a melhoria das condições do acesso da população aos serviços locais de saúde.

\section{O papel do empoderamento na relação profissional-paciente}

Sabe-se que o paciente com doença crônica, hipertensão e diabetes, necessita cuidar-se constantemente, ser corresponsável pela manutenção de sua saúde e qualidade de vida, gerir sua vida rodeada de cuidados com alimentação, atividade física, ingestão de medicamentos corretamente, assim como sua saúde mental. De tal forma, observamos que alguns pacientes aguardam o momento da consulta para externarem suas angústias relativas à doença, porém, em alguns momentos não conseguem espaço para o diálogo e a relação profissional-paciente não se concretiza, o que repercute negativamente na continuidade do tratamento.

A importância da relação clínica explica porque a adesão terapêutica depende mais do profissional de saúde do que das características pessoais do paciente. Em particular, o paciente é mais inclinado a corresponder à prescrição se pensa que conhece bem o profissional que o atende ${ }^{16}$. Assim, percebemos que a aderência ao autocuidado está intimamente ligada a essa relação, que deve ser baseada na confiança, no respeito aos anseios dos usuários, na escuta às suas necessidades, no estabelecimento de vínculo e autono$\mathrm{mia}^{17}$, o que possibilita a responsabilização (empoderamento), construção de propostas terapêuticas promotoras de saúde e, consequentemente, melhores resultados de saúde ${ }^{7}$.

Vivenciamos com os pacientes, no entanto, que, após conseguirem a consulta, os profissionais de saúde não davam a devida importância à pessoa que estava à sua frente com suas dúvidas e medos. Muitos relataram que a consulta "não passa de uma transcrição de receitas”. Narram que os profissionais não se mostram dispostos a tirar dúvidas nem a explicar procedimentos e cuidados a serem tomados.

Aqui não... só mesmo passam o remédio... quando você tem a primeira consulta ai eles verificam a pressão, tudo... passam o remédio... quando volta de novo que você quer falar com ele, ele evita... passa só aquele remédio que você já é acostumado a tomar né? Elas às vezes pega a minha receita que já vem, que já ta acostumada, copia e pronto! (Usuária 5)

Estas atitudes dos profissionais de saúde da atenção primária levam a interrogar: a qual modelo de atenção estão vinculados? Suas práticas indicam para um foco na conformidade, na qual a relação do profissional de saúde com o ser cuidado se reveste de autoridade que reduz a liberdade de escolha do paciente. Este agir clínico é considerado adequado para doenças agudas, porém, limitado para responder aos problemas de saúde; contrário, portanto, ao empoderamento, que propicia um processo de negociação levando em conta as necessidades, as expectativas e a cultura do paciente.

O encontro do profissional da saúde com o usuário é histórico, se dá a cada situação singular, não é possível ajustar-se a uma regra ou prescrição. Nele ocorre um encontro de escolha de valores de vida em busca da produção do comum, não cabe ao profissional só saber a necessidade do usuário, mas captar as capacidades dos indivíduos e comunidades na produção do comum como força propulsora para prosseguir a vida reinventando novos modos de viver ${ }^{18}$. O empoderamento neste sentido pode propiciar que renormalizações ocorram e os profissionais podem, assim, questionar os níveis de governança e é favorecido também o repensar das políticas de saúde.

Transformar práticas de saúde, portanto, exige mudanças no processo de construção dos sujeitos envolvidos no trabalho em saúde. Apenas com profissionais e usuários protagonistas e corresponsáveis é possível efetivar a aposta que o SUS faz na universalidade do acesso, na integralidade do cuidado e na equidade das ofertas em saúde. Requer, assim, novos sujeitos implicados em novas práticas de saúde. Pensar a saúde como experiência de criação de si e de modos de viver é tomar a vida em seu movimento de produção de normas e não de sujeição a elas ${ }^{19}$.

Uma vez que a doença crônica exige um tratamento permanente, faz-se necessário que o indivíduo cultive hábitos e atitudes que promovam a consciência para o autocuidado. Portanto, aderir ao tratamento é imprescindível para o controle da condição crônica e o sucesso da terapia proposta. 
A adesão ao tratamento está relacionada a fatores comportamentais, como percepção e formas de enfrentamento das adversidades, assim como a fatores externos, tais como problemáticas de vida e redes de apoio ${ }^{20}$. A vivência de cada usuário interfere na maneira de visualizar sua doença em seu contexto de vida, e, desta forma, também em como ele participa no tratamento. Por isso, é necessário sensibilidade ao profissional de saúde para perceber cada indivíduo como único e, desta maneira, adaptar sua forma de assistência para cada pessoa. Deve colocar como foco de sua atenção a pessoa e não a doença, transformando a relação de cuidado na medida em que o indivíduo se torna sujeito ativo de seu tratamento ${ }^{21}$.

Assim, compreender a percepção desses usuários sobre sua realidade, experiências vividas, promover o empoderamento e, até mesmo, o que consideram cuidado de si, é importante para que os profissionais procedam na conduta terapêutica embasados, aumentando, assim, as chances de negociação e elaboração de plano de ação conjunto ao tratamento. Na verdade, o paciente é o único e verdadeiro arquiteto de sua própria saúde e bemestar. O problema não é se ele controla a própria doença, mas como consegue gerenciar sua vida apesar da enfermidade. O empoderamento do paciente e o autocuidado são, portanto, a forma mais eficaz para lidar com as doenças crônicas, permitindo que o paciente tome uma consciência crítica em relação a seus problemas de saúde.

Deve-se alertar para o fato de que algumas pessoas do estudo utilizam terapias populares para cuidar-se e muitas vezes determinam maior interesse a tais práticas do que às indicações de medicamentos. Cabe ao profissional de saúde o papel de conhecer e respeitar tais práticas, mantendo uma relação de confiança onde a cultura do usuário não seja ignorada. Os profissionais não podem negar a cultura popular, nem descuidar do acompanhamento e controle das condições biofisiológicas dos pacientes, de forma a intervir antes que consequências mais graves sejam instaladas ${ }^{22}$. No entanto, o que percebemos foi que os profissionais de saúde não levam em conta tais conhecimentos populares, muitas vezes condenando-os e isso faz com que a relação profissional-paciente torne-se vulnerável.

Visualizar o indivíduo de forma diferenciada da preconizada no modelo convencional permite ao profissional de saúde o desempenho de uma assistência com abordagem integral. Dessa forma, sua ação deixa de limitar-se à cura de doenças ou ao tratamento de sintomatologias e passa a contribuir para um melhor desempenho nas questões referentes ao processo saúde-doença e, consequentemente, na qualidade de vida do usuário e das comunidades.

Muitos profissionais vêm descobrindo a grande potencialidade da relação dialogada profunda com seus pacientes e com os grupos comunitários locais para a reorganização das práticas de assistência e promoção da saúde, mas vão descobrindo que não basta querer se aproximar e dialogar. O diálogo entre o profissional de saúde e a população é difícil. Há muitos bloqueios culturais, distanciamentos pela desigualdade de poder e desentendimentos. A educação popular gera práticas de atenção e de promoção à saúde extremamente inovadoras e eficazes para quem busca a integralidade e a justiça social ${ }^{23}$.

\section{Prática educativa e empoderamento na relação de cuidado}

A educação destina-se a formar a consciência crítica e a autonomia. Requer a escuta ativa e o diálogo aberto e igual, já que o objetivo final da educação não é apenas uma compreensão da informação, mas incentivar as pessoas a definir os seus próprios problemas, encontrar as soluções para si e lidar com eles de forma eficaz, mesmo sob o aspecto emocional ${ }^{24}$.

No cotidiano das UBS do município de Fortaleza não há espaço para o desenvolvimento de ações de educação em saúde, pois, priorizam-se medidas clínicas assistenciais. Sabe-se que algumas doenças crônicas, como hipertensão e diabetes, dentre outras, requerem uma intervenção constante não só medicamentosa, mas também mediante troca de informações, acompanhamentos e orientações junto à comunidade, para que se consiga maior resolutividade no tratamento, na cura e eliminação, facetas estas, cabíveis, por intermédios de ações de "educação em saúde" integradas ao dia a dia da clínica.

No entanto, o espaço pertinente para seu desenvolvimento é mínimo na realidade das ações assistenciais neste cenário da atenção primária. Assim, tanto os gestores como os profissionais de saúde não demonstram interesse em "perder tempo" com esse tipo de atividade, procedimento absorvido pela maioria dos integrantes da comunidade, que não percebem uma intervenção educativa como assistência à saúde, preferindo o método tradicional de consulta e prescrição medicamentosa.

Os usuários que nunca tiveram contato com práticas de educação em saúde não reconhecem 
tal atividade como modificadora de sua realidade. No entanto, apreciariam se seus sentimentos e dúvidas fossem considerados durante a consulta. Já aqueles que obtiveram a oportunidade de experimentar essas práticas sentem-se motivados a cuidarem de si, e a multiplicar esses conhecimentos.

Quando a gente vai pra consulta os doutor não tem tempo de olhar nem pra gente, quem dirá de escutar nossos medos, nossas dúvidas, mas aqui no grupo não, a gente fala o que pensa, o que vive e entende melhor das coisas, ai a gente se sente importante, passa a querer ensinar os outros, a cuidar da gente, a viver melhor... (Usuária 12).

Assim, quando a situação dos atores sociais envolvidos na relação terapêutica não é compreendida, infere-se erroneamente acerca dos motivos que levam os usuários a não desempenharem práticas de autocuidado. Os profissionais de saúde parecem não reconhecer os pacientes como capazes de realizar seu próprio cuidado, deixando, assim, de orientá-los, informá-los e de realizar praticas de educação em saúde que levem ao empoderamento em sua interação clínica. Diante disso, perdem a oportunidade de conhecer histórias de vida, assim como os problemas vivenciados, causando grandes lacunas na relação profissional-paciente.

Ao conhecer a realidade do usuário e analisar sua fala, percebemos que múltiplos fatores impedem o empoderamento, pois as relações estabelecidas não favorecem que o mesmo tenha $o$ domínio sobre sua vida ${ }^{25}$, embora, muitas vezes, deseje ser ativo na interação do cuidado não encontra subsídios para fazê-lo.

Diante do que foi discutido, o fator educação, no que se refere à dificuldade de promoção do empoderamento do paciente para assumir responsabilidade pelas decisões sobre sua vida e saúde, foi apreendido por nós como o principal desencadeador dos outros aspectos que inibem ações de autocuidado. Assim, os próprios usuários relatam que o "não conhecimento acerca de sua doença", assim como "a gravidade" e as possíveis "complicações" levam-nos a não seguir as orientações sugeridas pelos profissionais.

Faz-se necessária a efetiva participação de todos os profissionais inseridos na ESF em uma prática educativa na atenção dispensada ao usuário. Tal atividade, além de orientar e tirar dúvidas, estimula a confiança na relação profissional-paciente, assim como torna os usuários indivíduos conscientes e corresponsáveis pelo seu tratamento. $\mathrm{O}$ ensino do cuidado de si é um processo importante, pois ajuda o indivíduo na ampliação do conhecimento do processo saúdedoença, aprimorando a autopercepção e beneficiando a mudança de hábitos necessária ${ }^{25}$.

Um dos caminhos para o desenvolvimento do cuidado permanente é a abordagem do usuário enquanto cidadão e protagonista. $\mathrm{O}$ autocuidado evidencia-se de forma relacional, sendo que paciente e profissional dependem um do outro para realizar o cuidado. Nesta vertente, o conhecimento e o acesso às informações são imprescindíveis para a realização de escolhas, e nisto incluem-se as práticas de empoderamento, que podem atender à estratégia básica para a promoção da saúde, considerada como tecnologia educacional inovadora.

O cuidado empoderador surge como uma atividade intencional que permite à pessoa adquirir conhecimento de si mesmo e daquilo que a cerca, podendo exercer mudanças nesse ambiente e na sua própria conduta. Além disso, capacita o doente crônico a definir os seus próprios problemas e necessidades, a compreender como pode resolver esses problemas com os seus recursos ou com apoios externos, e a promover ações mais apropriadas para fomentar uma vida saudável e de bem-estar' ${ }^{26}$.

É importante visualizar que trabalhar com saúde da família exige a análise acurada do contexto socioeconômico e cultural em que esta se insere, suas representações perante a sociedade, desvendar o entendimento da família, para que o conhecimento se consolide na prática, de forma a superar limites e possibilidades para a concretização das propostas terapêuticas ${ }^{22}$.

Por fim, os usuários sugerem a construção de espaços de bem-estar com profissionais qualificados que orientem práticas de exercícios físicos, além de atividades que promovam o relaxamento e a integração entre pessoas da comunidade. Relatam também que a revitalização de praças e parques públicos seria um grande incentivo para a realização de práticas de autocuidado.

Aprendemos com essas falas que a melhor forma de incentivar o autocuidado é com a aproximação da equipe com a comunidade. Somente conhecendo suas particularidades e suas necessidades é que a ESF poderá adequar sua conduta e, assim, alcançar seus objetivos.

A pessoa esclarecida, familiarizada de sua condição de ser com doença crônica, envolvida em seu tratamento tende a sentir-se mais segura e esperançosa quanto à evolução e prognóstico de sua doença. Assim, os profissionais de saúde, ao trabalharem com doentes crônicos, devem ter em mente que o cuidado é uma via de mão dupla, em 
que o ensinar e o aprender são construídos em bases de reciprocidade, entre pessoas e saberes ${ }^{21}$.

\section{Considerações finais}

As informações coletadas nos revelaram o importante papel que o profissional de saúde desempenha no empoderamento do usuário para um cuidado promotor de saúde. A sua atuação perpassa as demais dificuldades encontradas, no sentido de orientar as medidas necessárias e cabíveis que cada usuário e/ou comunidade poderá realizar de acordo com a sua realidade.

Desta forma, torna-se necessário reestruturar a conduta dos profissionais inseridos na Estratégia Saúde da Família, pois é função primordial promover a saúde, tanto individual como da coletividade. Assim, com esse intuito é essencial uma prática em saúde humanizada, focada também nos seus determinantes sociais. Deve-se repensar a Promoção da Saúde em uma lógica intersetorial (saúde, urbanização, transporte e segurança) e interdisciplinar. Somente conhecendo de perto os usuários, o contexto, as potencialidades e as adversidades da população atendida é que os profissionais irão manter uma relação profissionalpaciente que propicie o empoderamento.
Sabe-se que a prática do autocuidado é focada em uma relação dialógica, na qual profissionais e pacientes devem compreender que seu sucesso depende da negociação partilhada. Faz-se necessário, portanto, que os profissionais desenvolvam uma prática pautada na interação e na troca de experiências entre os integrantes da equipe de saúde, com vistas a oferecer uma assistência integral, que valorize o autocuidado como parte da vida, necessário ao bem-estar e desenvolvimento humano.

Acrescente-se ainda que as decisões e o compromisso dos gestores têm papel fundamental na qualidade do cuidado no que diz respeito à melhoria do acesso a UBS, ambientação das praças públicas, maior policiamento nas ruas, além de proporcionar espaços e incentivos à participação e educação popular.

Por fim, ao pensar um novo modelo de assistência ao doente crônico torna-se necessário reestruturar a qualificação dos profissionais inseridos na Estratégia Saúde da Família, inserir o empoderamento como pilar da sua prática de cuidado, uma vez que têm como função a promoção da saúde, em uma lógica intersetorial e interdisciplinar.

\section{Colaboradores}

PS Taddeo, KWL Gomes, A Caprara, AMA Gomes, GC Oliveira e TMM Moreira participaram igualmente de todas as etapas de elaboração do artigo. 


\section{Referências}

1. Freitas MC, Mendes MMR. Condição crônica: Análise do Conceito no contexto da saúde do adulto. Rev Latino-am Enfermagem 2007; 15(4):590-597.

2. Organização Mundial da Saúde (OMS). Cuidados inovadores para condições crônicas: componentes estruturais de ação. Brasília: OMS; 2003.

3. Bodenheimer T, Wagner EH, Grumbach K. Improving Primary Care for Patients with Chronic Ilness JAMA 2002; 288(14):1775-1779.

4. Marceca M, Ciccarelli S. Cronico. Care 2007; 3:3236.

5. Sousa JC. A doença crônica e o desafio da comorbilidade. Rev. Port. Clin. Geral 2007; 23:353-358.

6. Feste C, Anderson EM. Empowerment: from philosophy to practice. Patient Education Counselling 1995; 26(1-3):139-144.

7. Wagner EH. Chronic disease management: what will take to improve care for chronic illness? Effective Clinical Practice 1998; 1(1):2-4.

8. Ferreira V, Machado P. O programa informativo $N U D^{\star} I S T$ : análise qualitativa de informação escrita [dissertação]. Florianópolis: Universidade Federal de Santa Catarina; 1999.

9. Brasil. Ministério da Saúde. Conselho Nacional de Saúde. Resolução nº. 196/1996. Diretrizes e Normas Regulamentadoras de Pesquisas Envolvendo Seres Humanos. Diário Oficial da União 1996; out 10.

10. Travassos C, Martins M. Uma revisão sobre os conceitos de acesso e utilização de serviços de saúde. Cad Saude Publica 2004; 20(Supl. 2):S190-S198.

11. Brasil. Ministério da Saúde (MS). Secretaria de Gestão Estratégica e Participativa. Departamento de Apoio à Gestão Participativa. Políticas de promoção da equidade em saúde. Brasilia: MS; 2012. (Série B. Textos Básicos de Saúde)

12. Epping-Jordan JE, Pruitt SD, Bengoa R, Wagner EH. Developing research and practice. Improving the quality of health care for chronic conditions. Qual Saf Health Care 2004; 13:299-305.

13. Brasil. Ministério da Saúde (MS). Secretaria de atenção à saúde. Departamento de Atenção Básica. Política Nacional de Atenção Básica. Brasília: MS; 2006.

14. Organização Panamericana de Saúde (OPAS). Carta de Otawa. Primeira conferência Internacional sobre promoção de saúde. OPAS; 1986. [site na Internet]. [acessado 2012 mar 15].Disponível em: http:// www.opas.org.br/promocao/uploadArq/Ottawa.pdf

15. Becker D, Edmundo K, Nunes NR, Bonato D, Souza R. Empowerment e avaliação participativa em um programa de desenvolvimento local e promoção da saúde. Cien Saude Colet 2004; 9(3):655-667.

16. Dixon M, Sweeney K. The human effect in medicine: theory, research and practice. Oxford: Radcliffe Medical Press; 2000.
17. Campos GWS. A clínica do sujeito: por uma clínica reformulada e ampliada. In: Campos GWS, organizador. Saúde Paidéia. São Paulo: Hucitec; 2003. p. 51-67.

18. Brasil. Ministério da Saúde (MS). Secretaria-Executiva. Núcleo Técnico da Política Nacional de Humanização. HumanizaSUS: a clínica ampliada. Brasília: MS; 2004.

19. Pereira E, Barros R. Dicionário da Educação Profissional em Saúde. Fiocruz; 2009. [site na Internet]. [acessado 2011 out 11]. Disponível em: http://www.epsjv. fiocruz.br/dicionario/verbetes/hum.html

20. Maldaner CR, Beuter M, Brondani CM, Budó MLD, Pauletto MR. Fatores que influenciam a adesão ao tratamento na doença crônica: o doente em terapia hemodialítica. Rev Gaúcha Enferm 2008; 29(4):647653.

21. Silveira LMC, Ribeiro VMB. Grupo de adesão ao tratamento: espaço de "ensinagem" para profissionais de saúde e pacientes. Interface Comun Saúde Educ 2005; 9(16):91-104.

22. Lopes MCL, Carreira L, Marcon SS, Souza AC, Waidman MAP. O autocuidado em indivíduos com hipertensão arterial: um estudo bibliográfico. Revista Eletrônica de Enfermagem [Internet]. 2008; 10(1):198211. [acessado 2012 set 30]. Disponível em: http:// www.fen.ufg.br/revista/v10/n1/v10n1a18.htm

23. Vasconcelos SM, Bosi ML, Pontes RJS. Dimensões da qualidade na avaliação em saúde: concepções de gestores. Rev Saude Publica 2010; 44(2):318-324

24. Freire P. Pedagogia do Oprimido. $45^{\text {a }}$ Edição. São Paulo: Paz e Terra; 2005.

25. Leopardi MT. Teoria e método em assistência de enfermagem. 2a Edição. Florianópolis: Soldasoft; 2006.

26. Hammerschmidt KSA, Lenardt MH. Tecnologia Educacional Inovadora para o emponderamento junto a idosos com Diabetes Mellitus. Texto Contexto Enferm 2010; 19(2):358-365.

Artigo apresentado em 30/04/2012

Aprovado em 17/07/1012

Versão final apresentada em 30/08/2012 